\title{
Mesh Phase Analysis of Encased Differential Gear Train for Coaxial Twin-Rotor Helicopter
}

\author{
Donglin Zhang $\mathbb{D}^{1},{ }^{1}$ Rupeng Zhu ${ }^{(D)},{ }^{1}$ Bibo Fu, ${ }^{2}$ and Wuzhong Tan ${ }^{2}$ \\ ${ }^{1}$ National Key Laboratory of Science and Technology on Helicopter Transmission, Nanjing University of Aeronautics and Astronautics, \\ Nanjing 210016, China \\ ${ }^{2}$ AECC Hunan Aviation Powerplant Research Institute, Zhuzhou 412002, China
}

Correspondence should be addressed to Rupeng Zhu; rpzhu@nuaa.edu.cn

Received 15 April 2019; Accepted 14 July 2019; Published 25 July 2019

Academic Editor: Vasilios Spitas

Copyright (C) 2019 Donglin Zhang et al. This is an open access article distributed under the Creative Commons Attribution License, which permits unrestricted use, distribution, and reproduction in any medium, provided the original work is properly cited.

\begin{abstract}
Dynamic excitation caused by time-varying meshing stiffness is one of the most important excitation forms in gear meshing process. The mesh phase relations between each gear pair are an important factor affecting the meshing stiffness. In this paper, the mesh phase relations between gear pairs in an encased differential gear train widely used in coaxial twin-rotor helicopters are discussed. Taking the meshing starting point where the gear tooth enters contact as the reference point, the mesh phase difference between adjacent gear pairs is analyzed and calculated, the system reference gear pair is selected, and the mesh phase difference of each gear pair relative to the system reference gear pair is obtained. The derivation process takes into account the modification of the teeth, the processing, and assembly of the duplicate gears, which makes the calculation method and conclusion more versatile. This work lays a foundation for considering the time-varying meshing stiffness in the study of system dynamics, load distribution, and fault diagnosis of compound planetary gears.
\end{abstract}

\section{Introduction}

Planetary gear transmission is widely used in aviation, automobile and other industrial fields because of its strong loadcarrying capacity and small size. However, the vibration and noise caused by the dynamic meshing force and dynamic supporting force of the system affect its reliability and service life. Equally distributing the load to each planet and avoiding excessive vibration are the goals of design and manufacture of planetary gear train. The mesh phase relations between gear pairs are an important factor affecting the meshing stiffness of the system. It has a great influence on the load distribution and vibration of the system and has always been the focus of researchers.

Based on an improved potential energy method, a timevarying mesh stiffness calculation model considering crack growth was proposed. The meshing stiffness of normal gear pair and gear pair with different levels of crack is calculated by traditional method, proposed method, and ANSYS method, respectively. The influence of the crack level of the gear on the dynamic response was studied by using a one-stage gear system dynamic model [1]. Cui calculated the gear tooth thickness decrements by straight-affecting line method and parabolic-affecting line method. Based on the universal tooth profile equation, the meshing stiffness of gear pairs with different crack levels was calculated, and their effects on the vibration response are studied by fault detection indicators [2]. Considering three possible contact situations, the meshing stiffness under different torques is calculated and compared with the stiffness obtained by ISO 6336 . The results of internal and external meshing stiffness calculated by various models are compared [3]. Based on the potential energy principle, Chen deduced the stiffness calculation model considering the modification and calculated the stiffness of spur gears under different modification coefficients [4].

Kahraman established a lumped parameter dynamic model of a single-stage planetary gear train and obtained the natural modes and forced vibration response caused by static transmission error. The influence of mesh phase relation 
on the dynamic characteristics of four-planet system was studied [5]. Kahraman also established a nonlinear timevarying dynamic model of planetary transmission considering tooth profile error and time-varying meshing stiffness, and analyzed the influence of errors on dynamic load-sharing coefficient [6]. Based on the harmonic decomposition of meshing forces acting on the sun-planet and ring-planet mesh, Parker and Abbasha studied the suppression of mesh phase relations on different vibration types of planetary gears. The nonlinear dynamic characteristics of system considering mesh phase difference are studied by using analytical model and finite element model $[7,8]$. The results show that the rules of suppressing the vibration caused by mesh phase difference are applicable to the nonlinearity caused by contact loss of gear teeth [9].

Parker defined the mesh tooth number function to describe the mesh phase relations, deduced the calculation method of the phase difference between the gear pairs, and calculated the phase difference of each mesh gear pair in the single-stage planetary gear train of $\mathrm{OH} 58$ helicopter main reducer when the planet gear rotates in the positive and negative directions [10]. Chen Yong studied the relationship between mesh phase difference and torsional vibration and carried out experimental research under different rotational speeds and loads. The results show that the design considering mesh phase difference can effectively reduce the vibration and noise levels of planetary gear system, and the tooth profile contact ratio has no significant effect on the vibration and noise levels in the presence of phase differences [11].

Guo and Parker [12] proposed a method to accurately define the phase difference of the compound planetary gear system. By introducing the concepts of relative phase and reference datum, the mesh phase relations between any two gear pairs in a compound planetary gear train were derived. Wang [13] studied the influence of mesh phase on the vibration of spur planetary ring gear by using the superposition principle and Fourier series method and analyzed the relationship between modal characteristics and mesh phase difference. Gawande measured the noise level of the planetary gears and found that the noise level of the planetary gears with mesh phase difference was significantly lower than that of the planetary gears without mesh phase difference $[14,15]$.

Chaari [16] established a dynamic model of a single-stage planetary gear train with consideration of the mesh phase. The dynamic responses of healthy planetary gear train and planetary gear train containing defective gear tooth were compared in time and frequency domains, and the validity of Wigner-Ville method for fault diagnosis of planetary gear train was verified. $\mathrm{Li}[17,18]$ studied the mesh phase relations of two-stage planetary gears with meshed-planet gears. On this basis, the time-varying meshing stiffness was calculated, and the simulation analysis and experiment of gear tooth fault signal were compared. $\mathrm{Hu}$ added the mesh phase difference introduced by helical angle to that of spur gear to obtain the mesh phase difference of the helical gear, established a load sharing calculation model considering the mesh phase, and calculated the load sharing factors of the system under different phase relations. The trajectory of the sun gear

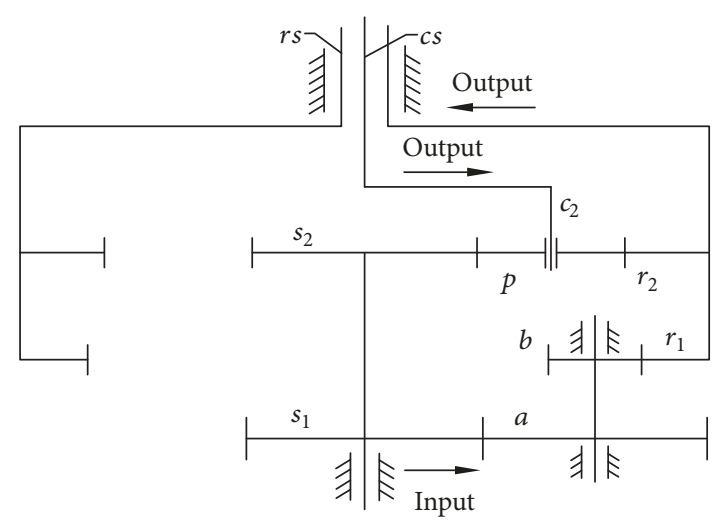

FIGURE 1: Configuration of encased differential planetary gear train.

[19] was obtained, which is in good agreement with the experimental results $[20,21]$.

In these literatures, pitch points are used as reference points of mesh phase difference, and researches are mainly for the single-stage planetary gear systems. There is no specific calculation method for the mesh phase difference of the compound planetary gears with stepped-planet gears. In this paper, the starting point of the meshing is taken as the reference point to make the calculation of phase difference more concise. The calculation method of phase difference of the compound planetary gear train with stepped-planet gears and duplicate central gears is derived. The gear modification is considered in the derivation process, which makes the conclusions in this paper more versatile.

\section{Definition of Mesh Phase Difference}

The research object of this paper is an encased differential planetary gear train shown in Figure 1 which can be regarded as a combination of two different subsystems: the fixed-shaft gear train with stepped-planet gears, that is, the encased stage, and the differential planetary gears is the differential stage. The input power is split into two paths as it is transmitted to the system: one is transmitted to the ring gear $r_{1}$ through the encased stage and the other is transmitted to the carrier $c_{2}$ and gear ring $r_{2}$ through the differential stage. When the number of teeth of each gear in a planetary gear train conforms to a specific relationship, the output shafts $c s$ and $r s$ can be output in reverse direction at the same rotational speed. The number of stepped planets $a b$ and planets $p$ is $M$ and $N$, respectively.

The gear pair sun gear $s_{1}$ and stepped gear $a_{i}(i=1,2, \ldots$, $M)$ is defined as $s_{1} a_{i}$, and the definitions of other gear pairs in the system are similar. The angular velocity of the component $h$ is $\omega_{h}\left(h=s_{1}, s_{2}, r_{1}, r_{2}, c_{2}, a, b, p\right)$ and the counterclockwise is positive, and the mesh period of all gear pairs is determined by the following:

$$
\begin{aligned}
& T_{s_{1} a_{i}}=\frac{2 \pi}{\omega_{s_{1}} z_{s_{1}}}=-\frac{2 \pi}{\omega_{a} z_{a}} \\
& T_{r_{1} b_{i}}=-\frac{2 \pi}{\omega_{r_{1}} z_{r_{1}}}=-\frac{2 \pi}{\omega_{b} z_{b}}
\end{aligned}
$$




$$
\begin{aligned}
T_{s_{2} p_{j}}= & \frac{2 \pi}{\left(\omega_{s_{2}}-\omega_{c_{2}}\right) z_{s_{2}}} \\
T_{r_{2} p_{j}}=-\frac{2 \pi}{\left(\omega_{r_{2}}-\omega_{c_{2}}\right) z_{r_{2}}} & \\
& (i=1,2, \ldots, M, j=1,2, \ldots, N)
\end{aligned}
$$

As shown in Figure 2, $\gamma_{A}^{B}$ represents the mesh phase difference of the mesh $B$ relative to the mesh $A$ [10], where $0 \leq \gamma<1$ and $\varepsilon_{g}$ is the contact ratio of the mesh $g$. Starting from the reference position, the gear pair $g$ is in contact at the meshing starting point for the first time at $t=t_{g}$, and the mesh phase difference can be obtained.

$$
\gamma_{A}^{B}=\operatorname{dec}\left(\frac{t_{B}-t_{A}}{T_{B}}\right)
$$

where $\operatorname{dec}(x)=x-\operatorname{int}(x)$ is a fractional function and $\operatorname{int}(x)$ is the nearest integer less than $x$.

\section{Mesh Phase Relations of Encased Differential Gear Train}

The calculation process of mesh phase difference of the encased differential planetary gear train is shown in Figure 3.

The initial assembly position of the compound planetary gears is shown in Figure 4. In the figure, $M N$ is the theoretical line of action, $A$ is the meshing starting point, $P$ is the pitch point of the mesh, $E$ is the point where the gear tooth exits contact, $D$ is the intersection point of the reference tooth midline and the addendum circle, and the definitions of radius and angle are given in the Appendix.

The angle of the midline of the planet gear $b_{1}$ reference tooth relative to that of the planet gear $a_{1}$ reference tooth is $\varphi_{a b}$. In order to meet the assembly conditions, the relative angles of gears $b$ and $a$ of each stepped-planet gear need to be the same. The angle of the midline of the sun gear $s_{2}$ reference space relative to that of the sun gear $s_{1}$ reference space is $\varphi_{s_{1} s_{2}}$.

3.1. Calculation of Phase Difference between Adjacent Mesh. For the sun-planet mesh $s_{1} a_{1}$, the calculations follow from the geometry of Figure 4(a).

$$
\begin{aligned}
\angle A_{1} O_{2} C_{1} & =\angle A_{1} O_{2} N_{1}-\angle N_{1} O_{2} P_{1}-\angle P_{1} O_{2} C_{1} \\
& =\alpha_{a a}-\alpha_{a}^{\prime}-\left(\frac{s_{a}}{2 r_{a}}-\left(\theta_{a a}-\theta_{a}\right)\right)
\end{aligned}
$$

here

$$
\begin{aligned}
\alpha_{a a} & =\arccos \left(\frac{r_{b a}}{r_{a a}}\right) \\
\alpha_{a}^{\prime} & \left.=\arccos \left[\frac{\left(\left(\left(m_{a} z_{a}+m_{s_{1}} z_{s_{1}}\right) / 2\right) \cos \alpha_{a}\right)}{O_{1} O_{2}}\right)\right]
\end{aligned}
$$

where $\mathrm{O}_{1} \mathrm{O}_{2}$ is the actual center distance between the sun gear $s_{1}$ and the stepped-planet gear $a_{1}$.

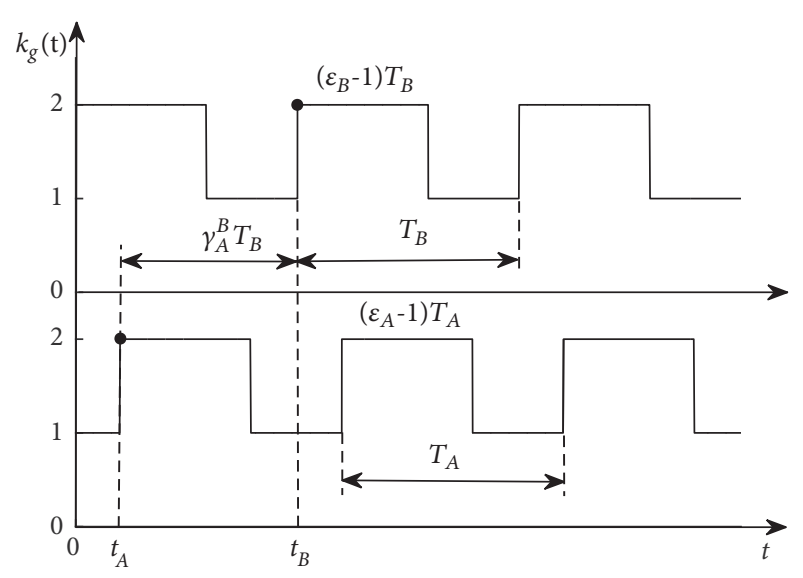

FIGURE 2: Schematic diagram of mesh phase difference.

It can be concluded that when the stepped-planet $a_{1}$ rotates from the initial position by an angle $\varphi_{a}$, the meshing starting point is in contact for the first time at $t=t_{1}$.

$$
\begin{aligned}
\varphi_{a} & =-\left[\frac{2 \pi}{Z_{a}}-\frac{2 \pi}{Z_{a}}\left(\operatorname{dec}\left(\frac{\angle A_{1} O_{2} C_{1}}{2 \pi / Z_{a}}\right)\right)\right] \\
t_{1} & =t_{0}+\frac{\varphi_{a}}{\omega_{a}}
\end{aligned}
$$

3.1.1. Calculation of Phase Difference $\gamma_{s_{1} a_{1}}^{s_{2} p_{1}}$. In order to meet the assembly requirements, it is advisable to design the installation position of planet gear $p_{1}$ to be the same as that of the stepped-planet gear $a_{1}$ circumferentially; that is, the center line $\mathrm{O}_{1} \mathrm{O}_{2}$ coincides with the center line $\mathrm{O}_{3} \mathrm{O}_{4}$ in the circumferential direction, as shown in Figure 4(b).

For the sun-planet mesh $s_{2} p_{1}$, geometrical relation can be derived from Figures 4(b) and 4(c).

$$
\begin{aligned}
\angle A_{3} O_{4} C_{3}= & \angle A_{3} O_{4} P_{3}+\angle P_{3} O_{4} D_{3}-\angle D_{3} O_{4} C_{3} \\
= & \alpha_{a p}-\alpha_{s_{2}}^{\prime}+\varphi_{s_{1} s_{2}} \frac{z_{s_{2}}}{z_{p}} \\
& -\left(\frac{s_{p}}{2 r_{p}}-\left(\theta_{a p}-\theta_{p}\right)\right)
\end{aligned}
$$

here

$$
\begin{aligned}
& \left.\alpha_{s_{2}}^{\prime}=\arccos \left[\frac{\left(\left(\left(m_{p} z_{p}+m_{s_{2}} z_{s_{2}}\right) / 2\right) \cos \alpha_{p}\right)}{O_{3} O_{4}}\right)\right] \\
& \alpha_{a p}=\arccos \left(\frac{r_{b p}}{r_{a p}}\right)
\end{aligned}
$$

where $\mathrm{O}_{3} \mathrm{O}_{4}$ is the actual center distance between the sun gear $s_{2}$ and the planet gear $p_{1}$.

When the planet $p_{1}$ rotates from the initial position by an angle $\varphi_{p}$, the meshing starting point is in contact for the first time at $t=t_{2}$. 


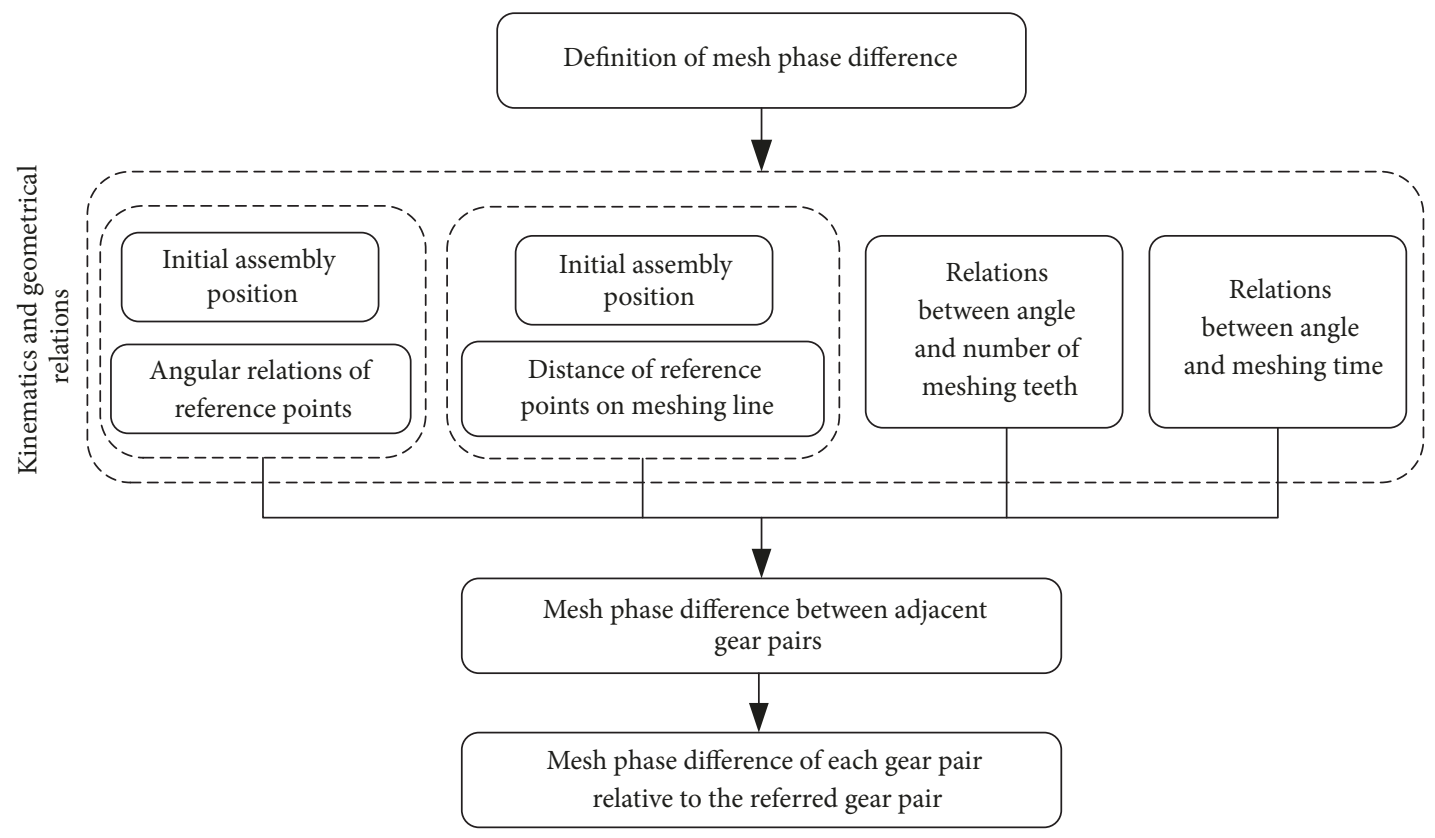

FIgURE 3: Flow chart of mesh phase difference calculation.

$$
\begin{aligned}
\varphi_{p} & =-\left[\frac{2 \pi}{Z_{p}}-\frac{2 \pi}{Z_{p}}\left(\operatorname{dec}\left(\frac{\angle A_{3} O_{4} C_{3}}{2 \pi / Z_{p}}\right)\right)\right] \\
t_{2} & =t_{0}+\frac{\varphi_{p}}{\omega_{p}-\omega_{c_{2}}}
\end{aligned}
$$

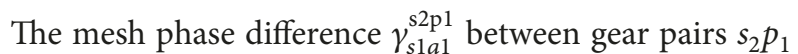
and $s_{1} a_{1}$ is

$$
\gamma_{s_{1} a_{1}}^{s_{2} p_{1}}=\operatorname{dec}\left(\frac{t_{2}-t_{1}}{T_{s_{2} p_{1}}}\right)
$$

3.1.2. Calculation of Phase Difference $\gamma_{s_{1} a_{1}}^{r_{1} b_{1}}$. As shown in Figure $4(\mathrm{a}), B_{2}$ is the starting point of the involute of the reference gear teeth on the base circle, and the curves $\widehat{B_{2} N_{2}}$ and $\mathrm{N}_{2} \mathrm{~A}_{2}$ are calculated as follows:

$$
\begin{aligned}
& \widehat{B_{2} N_{2}}=r_{b b} * \angle N_{2} O_{2} B_{2} \\
& N_{2} A_{2}=M_{2} A_{2}-M_{2} N_{2}=\sqrt{r_{a r_{1}}^{2}-r_{b r_{1}}^{2}}-O_{1} O_{2} \sin \alpha_{r_{1}}^{\prime}
\end{aligned}
$$

where

$$
\begin{aligned}
\angle N_{2} \mathrm{O}_{2} B_{2} & =\pi-\varphi_{a b}-N_{2} \mathrm{O}_{2} P_{2}-\angle B_{2} \mathrm{O}_{2} D_{2} \\
& =\pi-\varphi_{a b}-\alpha_{r_{1}}^{\prime}-\left(\frac{s_{b}}{2 r_{b}}+\theta_{b}\right)
\end{aligned}
$$

Equations (14)-(16) give

$$
\begin{aligned}
L_{r_{1} b}= & \widehat{B_{2} N_{2}}+N_{2} A_{2} \\
= & r_{b b} *\left(\pi-\alpha_{r_{1}}^{\prime}-\left(\frac{s_{b}}{2 r_{b}}+\theta_{b}+\varphi_{a b}\right)\right) \\
& +\sqrt{r_{a r_{1}}^{2}-r_{b r_{1}}^{2}}-O_{1} O_{2} \sin \alpha_{r_{1}}^{\prime}
\end{aligned}
$$

where

$$
\left.\alpha_{r_{1}}^{\prime}=\arccos \left[\frac{\left(\left(\left(m_{r_{1}} z_{r_{1}}-m_{b} z_{b}\right) / 2\right) \cos \alpha_{r_{1}}\right)}{O_{1} O_{2}}\right)\right]
$$

From the initial position, the meshing starting point of gear pair $r_{1} b_{1}$ is in contact for the first time at $t=t_{3}$.

$$
t_{3}=t_{0}+T_{r_{1} b_{1}} \operatorname{dec}\left(\frac{L_{r_{1} b}}{p_{b b}}\right)
$$

The mesh phase difference $\gamma_{s_{1} a_{1}}^{r_{1} b_{1}}$ between gear pair $r_{1} b_{1}$ and gear pair $s_{1} a_{1}$ is

$$
\gamma_{s_{1} a_{1}}^{r_{1} b_{1}}=\operatorname{dec}\left(\frac{t_{3}-t_{1}}{T_{r_{1} b_{1}}}\right)
$$

3.1.3. Calculation of Phase Difference $\gamma_{s_{2} p_{1}}^{r_{2} p_{1}}$. Figure 4(b) shows the following for length of the curves:

$$
\begin{aligned}
A_{3} N_{3} & =\sqrt{\left(O_{4} A_{3}\right)^{2}-\left(O_{4} N_{3}\right)^{2}}=\sqrt{r_{a p}^{2}-r_{b p}^{2}} \\
\widehat{N_{3} N_{4}} & =r_{b p} * \angle N_{3} O_{4} N_{4}=r_{b p} *\left(\pi-\alpha_{s_{2}}^{\prime}-\alpha_{r_{2}}^{\prime}\right) \\
N_{4} A_{4} & =M_{4} A_{4}-M_{4} N_{4} \\
& =\sqrt{r_{a r_{2}}^{2}-r_{b r_{2}}^{2}}-O_{3} O_{4} \sin \alpha_{r_{2}}^{\prime}
\end{aligned}
$$

According to the above equations (21)-(23), we have

$$
\begin{aligned}
L_{s_{2} r}= & A_{3} N_{3}+\widehat{N_{3} N_{4}}+N_{4} A_{4} \\
= & \sqrt{r_{a p}^{2}-r_{b p}^{2}}+r_{b p} *\left(\pi-\alpha_{s_{2}}^{\prime}-\alpha_{r_{2}}^{\prime}\right) \\
& +\sqrt{r_{a r_{2}}^{2}-r_{b r_{2}}^{2}}-O_{3} O_{4} \sin \alpha_{r_{2}}^{\prime}
\end{aligned}
$$




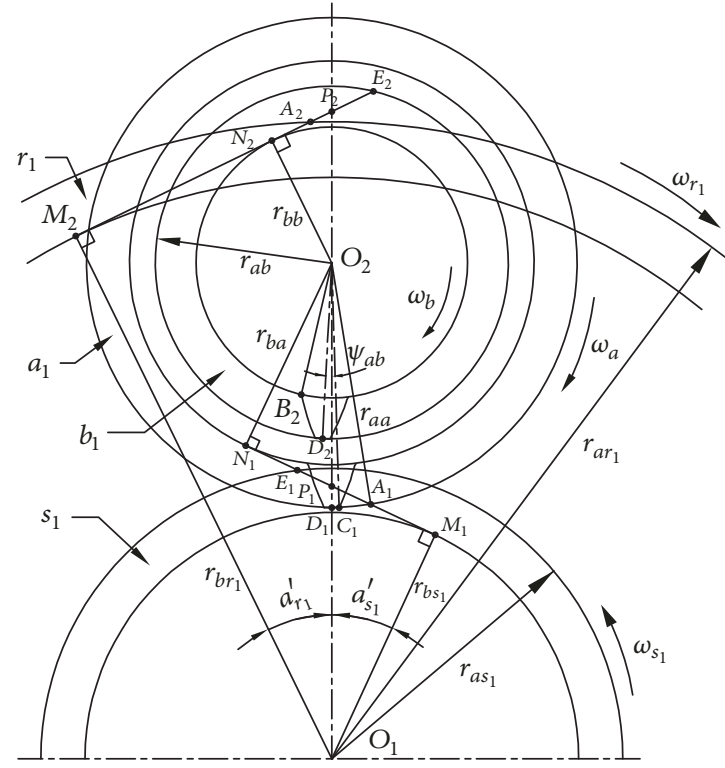

(a) Encased stage

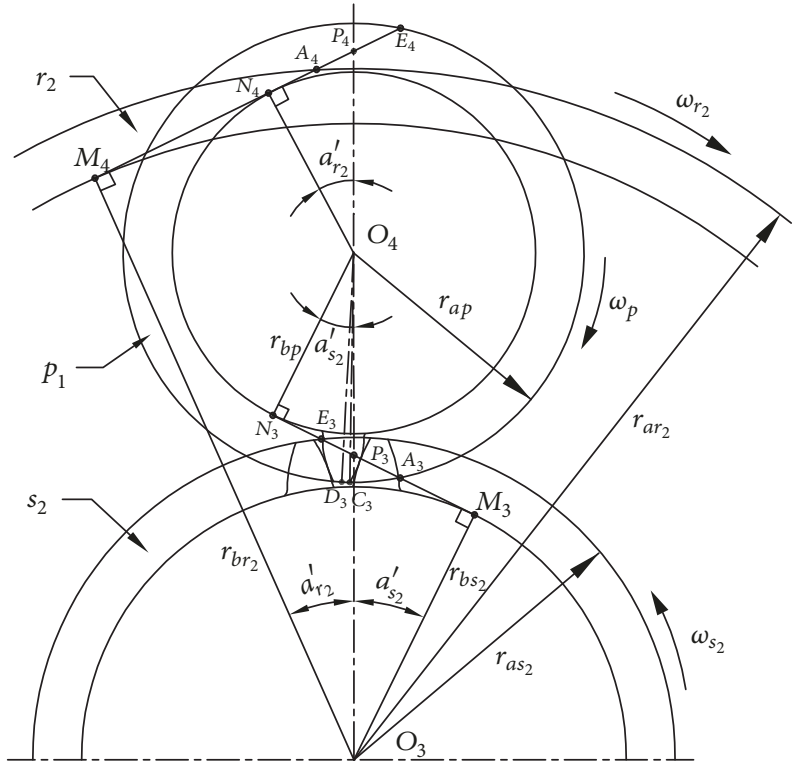

(b) Differential stage

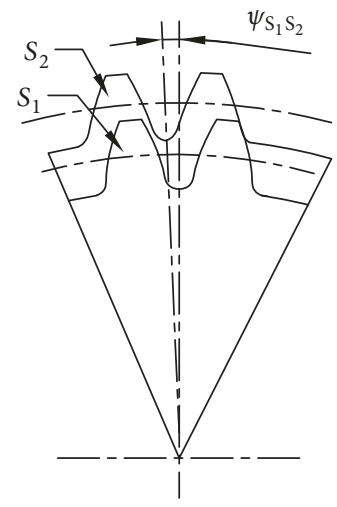

(c) Relative position of duplicate sun gears

FIGURE 4: Initial position of the planetary gear train.

The phase difference between internal gear pair and external gear pair of the differential stage is

$$
\gamma_{s_{2} p_{1}}^{r_{2} p_{1}}=\operatorname{dec}\left(\frac{L_{s_{2} r}-s_{b p}}{p_{b p}}\right)
$$

where the tooth thickness on the base circle $s_{b p}=(\pi / 2+$ $\left.2 x_{p} \tan \alpha_{p}\right) m_{p} \cos \alpha_{p}+2 r_{b p} \theta_{p}$.

3.1.4. Calculation of Phase Difference $\gamma_{s_{1}}^{r_{1} b_{i}}$. The number of teeth meshed when the sun gear $s_{1}$ rotates one revolution is $z_{s_{1}}$. When the sun gear $s_{1}$ rotates from the position of the stepped-planet gear $a_{1}$ to the position of the stepped-planet gear $a_{i}$, the angle of rotation $\varphi_{s_{1}}^{i}$ and the number of teeth meshed $z_{s_{1}}^{i}$ are

$$
\begin{aligned}
\varphi_{s_{1}}^{i} & =\frac{2 \pi}{M}(i-1) \\
z_{s_{1}}^{i} & =\frac{Z_{s_{1}}}{2 \pi} \varphi_{s_{1}}^{i}
\end{aligned}
$$

From (26) and (27), the mesh phase difference between gear pairs $s_{1} a_{i}$ and $s_{1} a_{1}$ is

$$
\gamma_{s_{1} a_{1}}^{s_{1} a_{i}}=\operatorname{dec}\left(\frac{z_{s_{1}}}{2 \pi} \varphi_{s_{1}}^{i}\right)=\operatorname{dec}\left(\frac{z_{s_{1}}}{M}(i-1)\right)
$$

3.1.5. Calculation of Phase Difference $\gamma_{r_{1}}^{r_{1} b_{1}}$. Similarly, when the ring gear $r_{1}$ rotates from the position of the planet $b_{1}$ to the position of the planet gear $b_{i}$, the angle of rotation $\varphi_{r_{1}}^{i}$ and the number of teeth meshed $z_{r_{1}}^{i}$ are as follows: 


$$
\begin{aligned}
\varphi_{r_{1}}^{i} & =2 \pi-\frac{2 \pi}{M}(i-1) \\
z_{r_{1}}^{i} & =\frac{Z_{r_{1}}}{2 \pi} \varphi_{r_{1}}^{i}
\end{aligned}
$$

The mesh phase difference between the gear pairs $r_{1} b_{i}$ and $r_{1} b_{1}$ obtained by (29) and (30) is

$$
\gamma_{r_{1} b_{1}}^{r_{1} b_{i}}=\operatorname{dec}\left(\frac{Z_{r_{1}}}{2 \pi} \varphi_{r_{1}}^{i}\right)=\operatorname{dec}\left(-\frac{Z_{r_{1}}}{M}(i-1)\right)
$$

3.1.6. Calculation of Phase Difference $\gamma_{s_{2} p_{1}}^{s_{2} p_{j}}$. It is known that the angle between the $j$-th planet gear and the 1st planet gear in the differential stage is

$$
\varphi_{j}=\frac{2 \pi}{N}(j-1)
$$

When the sun gear $s_{2}$ rotates over the angle $\varphi_{j}$ relative to the carrier $c_{2}$, the $j$-th planet gear moves to the initial position of the 1st planet gear, and the time required is

$$
t_{s j}=\frac{\varphi_{j}}{\omega_{s_{2}}-\omega_{c_{2}}}
$$

Considering that the time difference caused by mesh phase difference is $\Delta t_{s j}=\gamma_{s_{2} p_{1}}^{s_{2} p_{j}} T_{s_{2} p_{j}}$, the time $t_{s j}$ can also be expressed as

$$
\left(\gamma_{s_{2} p_{1}}^{s_{2} p_{j}}+C_{s_{2}}\right) T_{s_{2} p_{j}}=t_{s j}
$$

where $C_{s 2}$ is an integer. Available from (1), (33) and (34), the mesh phase difference between the gear pairs $s_{2} p_{j}$ and $s_{2} p_{1}$ is

$$
\gamma_{s_{2} p_{1}}^{s_{2} p_{j}}=\operatorname{dec}\left(\frac{z_{s_{2}}}{2 \pi} \varphi_{j}\right)=\operatorname{dec}\left(\frac{z_{s_{2}}}{N}(j-1)\right)
$$

3.1.7. Calculation of Phase Difference $\gamma_{r_{2} p_{1}}^{r_{2} p_{j}}$. Similar to the above, when the ring gear rotates $2 \pi-\varphi_{j}$ relative to the carrier $c_{2}$, the $j$-th planet gear moves to the initial position of the 1 st planet gear, and the time required is

$$
t_{r j}=-\frac{2 \pi-\varphi_{j}}{\omega_{r_{2}}-\omega_{c_{2}}}
$$

Considering that the time difference caused by mesh phase difference is $\Delta t_{r j}=\gamma_{r_{2} p_{1}}^{r_{2} p_{j}} T_{r_{2} p_{j}}$, we obtain the following:

$$
\left(\gamma_{r_{2} p_{1}}^{r_{2} p_{j}}+C_{r_{2}}\right) T_{r_{2} p_{j}}=\frac{-\left(2 \pi-\varphi_{j}\right)}{\omega_{r_{2}}-\omega_{c_{2}}}
$$

where $C_{r_{2}}$ is an integer. The mesh phase difference between the gear pairs $r_{2} p_{j}$ and $r_{2} p_{1}$ can be obtained by introducing (1) into (37).

$$
\gamma_{r_{2} p_{1}}^{r_{2} p_{j}}=\operatorname{dec}\left(\frac{z_{r_{2}}\left(2 \pi-\varphi_{j}\right)}{2 \pi}\right)=\operatorname{dec}\left(-\frac{z_{r_{2}}}{N}(j-1)\right)
$$

3.2. Calculation of Comprehensive Mesh Phase Difference. Through the above analysis, the mesh phase differences between adjacent gear pairs are obtained. In order to unify the time difference caused by mesh phase difference to the absolute time of the system, it is necessary to synthesize the adjacent phase difference to get the phase difference of each gear pair relative to the base referred mesh. In the following analysis, the mesh $s_{1} a_{1}$ is selected as the base referred mesh of the system.

3.2.1. Calculation of Phase Difference $\gamma_{s_{1} a_{1}}^{r_{2} p_{1}}$. After the contact of the meshing start point of sun-planet mesh $s_{2} p_{1}$, the time $t_{r 2 \mathrm{p} 1}^{\prime}$ at which the meshing start point of ring-planet mesh $r_{2} p_{1}$ contact for the first time is

$$
t_{r_{2} p_{1}}^{\prime}=\gamma_{s_{2} p_{1}}^{r_{2} p_{1}} T_{r_{2} p_{1}}+t_{s_{2} p_{1}}=\gamma_{s_{2} p_{1}}^{r_{2} p_{1}} T_{r_{2} p_{1}}+\gamma_{s_{1} a_{1}}^{s_{2} p_{1}} T_{s_{2} p_{1}}+t_{1}
$$

Relative to the base referred mesh $s_{1} a_{1}$, the mesh phase difference of the gear pair $r_{2} p_{1}$ is

$$
\gamma_{s_{1} a_{1}}^{r_{2} p_{1}}=\operatorname{dec}\left(\frac{t_{r_{2} p_{1}}^{\prime}-t_{1}}{T_{r_{2} p_{1}}}\right)=\operatorname{dec}\left(\gamma_{s_{2} p_{1}}^{r_{2} p_{1}}+\gamma_{s_{1} a_{1}}^{s_{2} p_{1}}\right)
$$

3.2.2. Calculation of Phase Difference $\gamma_{s_{1} a_{1}}^{r_{1} b_{i}}$. After the mesh $r_{1} b_{1}$ contacts at meshing starting point, the first contact time of meshing starting point of the mesh $r_{1} b_{i}$ is

$$
t_{r_{1} b_{i}}^{\prime}=\gamma_{r_{1} b_{1}}^{r_{1} b_{i}} T_{r_{1} b_{i}}+t_{r_{1} b_{1}}=\gamma_{r_{1} b_{1}}^{r_{1} b_{i}} T_{r_{1} b_{i}}+\gamma_{s_{1} a_{1}}^{r_{1} b_{1}} T_{r_{1} b_{1}}+t_{1}
$$

The mesh phase difference of the gear pair $r_{1} b_{i}$ relative to the base referred mesh $s_{1} a_{1}$ is

$$
\gamma_{s_{1} a_{1}}^{r_{1} b_{i}}=\operatorname{dec}\left(\frac{t_{r_{1} b_{i}}^{\prime}-t_{1}}{T_{r_{1} b_{i}}}\right)=\operatorname{dec}\left(\gamma_{r_{1} b_{1}}^{r_{1} b_{i}}+\gamma_{s_{1} a_{1}}^{r_{1} b_{1}}\right)
$$

3.2.3. Calculation of Phase Difference $\gamma_{s_{1} a_{1}}^{s_{2} p_{j}}$. After the mesh $s_{2} p_{1}$ contacts at meshing starting point, the first contact time of meshing starting point of the mesh $s_{2} p_{j}$ is

$$
t_{s_{2} p_{j}}^{\prime}=\gamma_{s_{2} p_{1}}^{s_{2} p_{j}} T_{s_{2} p_{j}}+t_{s_{2} p_{1}}=\gamma_{s_{2} p_{1}}^{s_{2} p_{j}} T_{s_{2} p_{j}}+\gamma_{s_{1} a_{1}}^{s_{2} p_{1}} T_{s_{2} p_{1}}+t_{1}
$$

The mesh phase difference of the gear pair $s_{2} p_{j}$ relative to the base referred mesh $s_{1} a_{1}$ is

$$
\gamma_{s_{1} a_{1}}^{s_{2} p_{j}}=\operatorname{dec}\left(\frac{t_{s_{2} p_{j}}^{\prime}-t_{1}}{T_{s 2 p j}}\right)=\operatorname{dec}\left(\gamma_{s_{2} p_{1}}^{s_{2} p_{j}}+\gamma_{s_{1} a_{1}}^{s_{2} p_{1}}\right)
$$

3.2.4. Calculation of Phase Difference $\gamma_{s_{1} a_{1}}^{r_{2} p_{j}}$. After the mesh $r_{2} p_{1}$ contacts at meshing starting point, the first contact time of meshing starting point of the mesh $r_{2} p_{j}$ is

$$
t_{r_{2} p_{j}}^{\prime}=\gamma_{r_{2} p_{1}}^{r_{2} p_{j}} T_{r_{2} p_{j}}+t_{r_{2} p_{1}}=\gamma_{r_{2} p_{1}}^{r_{2} p_{j}} T_{r_{2} p_{j}}+\gamma_{s_{1} a_{1}}^{r_{2} p_{1}} T_{r_{2} p_{1}}+t_{1}
$$

The mesh phase difference of the gear pair $r_{2} p_{j}$ relative to the base referred mesh $s_{1} a_{1}$ is

$$
\gamma_{s_{1} a_{1}}^{r_{2} p_{j}}=\operatorname{dec}\left(\frac{t_{r_{2} p_{j}}^{\prime}-t_{1}}{T_{r_{2} p_{j}}}\right)=\operatorname{dec}\left(\gamma_{r_{2} p_{1}}^{r_{2} p_{j}}+\gamma_{s_{1} a_{1}}^{r_{2} p_{1}}\right)
$$


TABLE 1: Encased differential planetary gear train parameters.

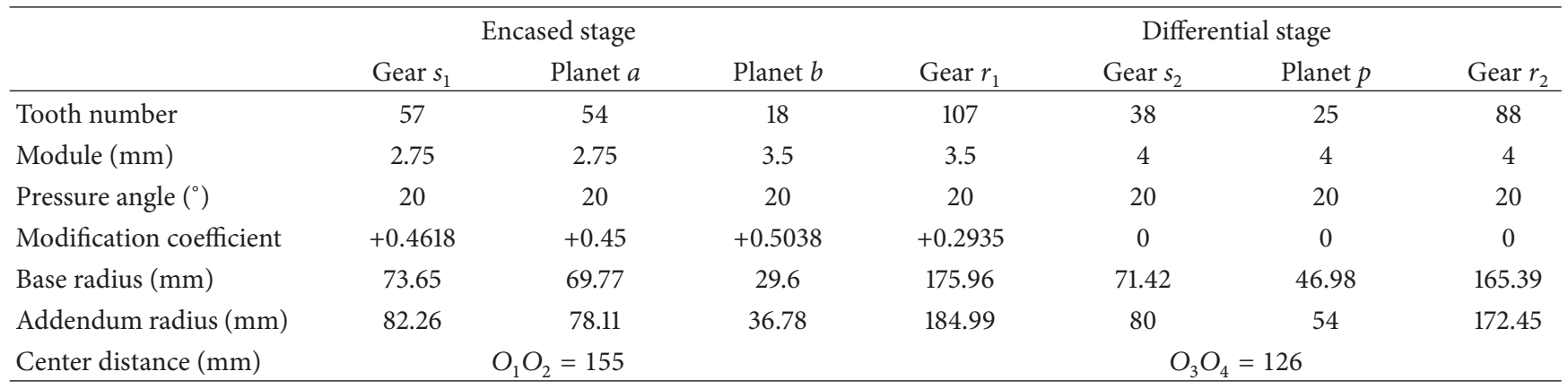

Since the starting point of the referred gear pair $s_{1} a_{1}$ contacts at $t=t_{1}$, the time at which the meshing starting point of the gear pair $g$ contacts for the first time is

$$
t_{g}=\gamma_{s_{1} a_{1}}^{g} T_{g}+t_{1}
$$

Then the mesh tooth variation function in the system can be expressed as [12]

$$
k_{g}(t)=\kappa_{g}\left(t-t_{g}\right)=\kappa_{g}\left(t-\gamma_{s_{1} a_{1}}^{g} T_{g}-t_{1}\right)
$$

where $\kappa_{g}(\tau)$ is a periodic time-varying mesh tooth variation function of the gear pair $g, \tau=0$ corresponds to the meshing starting point, and $t$ is absolute time of the system.

\section{Example Calculation of Phase Difference in Encased Differential Gear Train}

Table 1 shows the parameters of the encased differential planetary gear train with $M=6, N=6$. For the convenience of processing and assembly, it is usually guaranteed that the midline of referred gear tooth or space of the duplicate gear is coincident during machining, that is, the offset angle $\varphi_{s_{1} s_{2}}=$ $0, \varphi_{a b}=0$. The mesh phase difference of all gear pairs in the system relative to the referred gear pair $s_{1} a_{1}$ is calculated as shown in Table 2.

When the input shaft speed is $n_{s_{1}}=1490(\mathrm{r} / \mathrm{min})$, the mesh period of all gear meshes in the example system can be obtained.

$$
\begin{aligned}
& T_{s_{1} a_{i}}=7.06 \times 10^{-4}(\mathrm{~s}) \\
& T_{r_{1} b_{i}}=2.12 \times 10^{-3}(\mathrm{~s}) \\
& T_{s_{2} p_{j}}=T_{r_{2} p_{j}}=1.29 \times 10^{-3}(\mathrm{~s})
\end{aligned}
$$

Without loss of generality, let the starting time $t_{1}=0$ of the reference gear pair $s_{1} a_{1}$ be in contact. The gear pair $s_{2} p_{j}$ mesh tooth variation function changes with time as shown in Figure 5, and the other gear pairs in the system can be similarly unified to the system time $t$.

The time-varying meshing stiffness of each gear pair in a meshing period calculated by theoretical method or finite element method combined with the meshing time difference caused by the phase difference can be applied to the dynamic
TABLE 2: Comprehensive mesh phase difference.

\begin{tabular}{c} 
Mesh phase difference $\gamma$ \\
\hline$\gamma_{s_{1} a_{1}}^{s_{1} a_{1}}=0 \gamma_{s_{1} a_{1}}^{s_{1} a_{2}}=0.5000$ \\
$\gamma_{s_{1} a_{1}}^{s_{1} a_{3}}=0 \gamma_{s_{1} a_{1}}^{s_{1} a_{4}}=0.5000$ \\
$\gamma_{s_{1} a_{1}}^{s_{1} a_{5}}=0 \gamma_{s_{1} a_{1}}^{s_{1} a_{6}}=0.5000$ \\
$\gamma_{s_{1} a_{1}}^{r_{1} b_{1}}=0.1246 \gamma_{s_{1} a_{1}}^{r_{1} b_{2}}=0.2913$ \\
$\gamma_{s_{1} a_{1}}^{r_{1} b_{3}}=0.4579 \gamma_{s_{1} a_{1}}^{r_{1} b_{4}}=0.6246$ \\
$\gamma_{s_{1} a_{1}}^{r_{1} b_{5}}=0.7913 \gamma_{s_{1} a_{1}}^{r_{1} b_{6}}=0.9579$ \\
$\gamma_{s_{1} a_{1}}^{s_{2} p_{1}}=0.1978 \gamma_{s_{1} a_{1}}^{s_{2} p_{2}}=0.5311$ \\
$\gamma_{s_{1} a_{1}}^{s_{2} p_{3}}=0.8645 \gamma_{s_{1} a_{1}}^{s_{2} p_{4}}=0.1978$ \\
$\gamma_{s_{1} a_{1}}^{s_{2} p_{5}}=0.5311 \gamma_{s_{1} a_{1}}^{s_{2} p_{6}}=0.8645$ \\
$\gamma_{s_{1} a_{1}}^{r_{2} p_{1}}=0.0432 \gamma_{s_{1} a_{1}}^{r_{2} p_{2}}=0.3765$ \\
$\gamma_{s_{1} a_{1}}^{r_{2} p_{3}}=0.7099 \gamma_{s_{1} a_{1}}^{r_{2} p_{4}}=0.0432$ \\
$\gamma_{s_{1} a_{1}}^{r_{2} p_{5}}=0.3765 \gamma_{s_{1} a_{1}}^{r_{2} p_{6}}=0.7099$
\end{tabular}

model of the system, which can accurately describe the meshing stiffness of all gear pairs in the model at any time and carry out the dynamic analysis of the gear system.

\section{Conclusions}

The accurate solution of the mesh phase difference of all gear pairs is an extremely important part of the study of the dynamics, fault diagnosis, and load sharing characteristics of compound planetary gear train. In this paper, for the encased differential compound planetary gear train widely used in coaxial twin-rotor helicopters, taking the meshing starting point as the reference point, considering the gear modification, the calculation method of mesh phase difference is studied.

Firstly, the initial position of the system and the referred gear pair of the system are defined, and the time when the referred gear pair is in contact at the meshing starting point is calculated. According to the angle relations of the meshing starting points and the distance relations of the starting points 


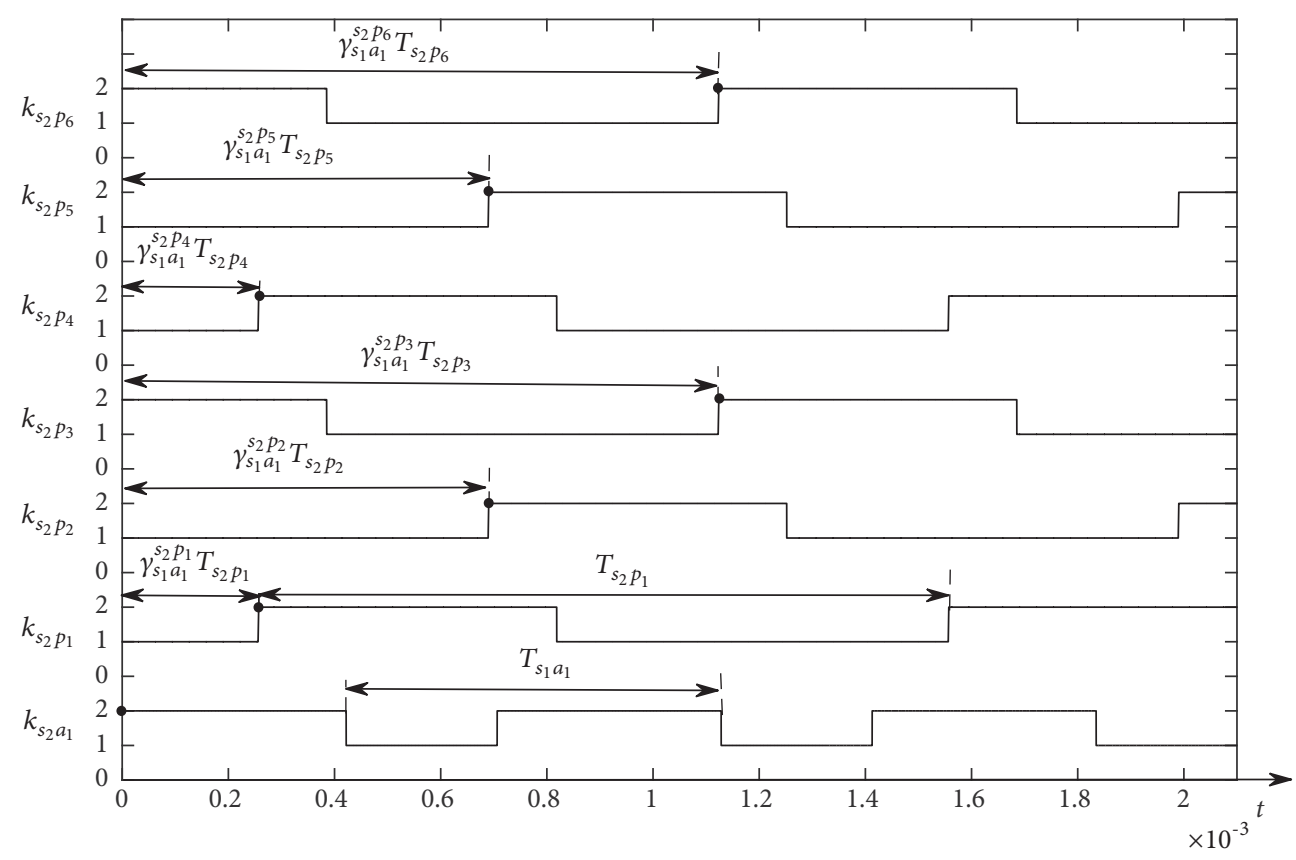

FigURE 5: Mesh tooth variation function of gear pairs $s_{2} p_{j}$.

on the meshing line, from the initial position, the time at which the gear pair adjacent to the base referred gear pair first contacted at the starting point is calculated, and the phase difference can be obtained by the meshing time difference between the gear pairs. The phase difference between planet gears is calculated by the relations between the number of meshing teeth and the meshing time caused by the symmetry of planetary gear train.

Based on the phase difference of adjacent gear pairs, the calculation method of the phase difference of each gear pair relative to the referred gear pair is derived. The derivation process considers the processing and assembly requirements of the duplicate gear, and a specific compound planetary gear train is calculated as an example, which lays a foundation for the accurate introduction of time-varying meshing stiffness into the dynamic analysis of compound planetary gear train.

\section{Nomenclature}

$z_{h}$ : Number of teeth of gear $h$

$\alpha_{a h}$ : Pressure angle on addendum circle of gear $h$

$\alpha_{h}^{\prime}$ : Working pressure angle of gear pair where central gear $h$ is located

$\theta_{h}:$ Involute function $\theta_{h}=\tan \alpha_{h}-\alpha_{h}$

$\theta_{a h}:$ Involute function $\theta_{a h}=\tan \alpha_{a h}-\alpha_{a h}$

$s_{h}$ : Tooth thickness at reference circle of gear $h$

$r_{h}$ : Reference circle radius of gear $h$

$r_{b h}$ : Base circle radius of gear $h$

$r_{a h}$ : Addendum circle radius of gear $h$

$p_{b h}$ : Base circle pitch of gear $h$

$s_{b h}$ : Tooth thickness at base circle of gear $h$

$x_{h}$ : Modification coefficient of gear $h$

$m_{h}$ : Modulus of the gear $h$.

\section{Data Availability}

The data used to support the findings of this study are available from the corresponding author upon request.

\section{Conflicts of Interest}

The authors declare that there are no conflicts of interest regarding the publication of this paper.

\section{Acknowledgments}

This work was supported by the National Natural Science Foundation of China [grant number 51775265].

\section{References}

[1] X. J. Zhou, Y. M. Shao, Y. G. Lei et al., “Time-varying meshing stiffness calculation and vibration analysis for a 16DOF dynamic model with linear crack growth in a pinion," Journal of Vibration and Acoustics, vol. 134, no. 1, Article ID 011011, 2012.

[2] L. Cui, H. Zhai, and F. Zhang, "Research on the meshing stiffness and vibration response of cracked gears based on the universal equation of gear profile," Mechanism and Machine Theory, vol. 94, pp. 80-95, 2015.

[3] M. Iglesias, A. Fernandez del Rincon, A. de-Juan, A. DiezIbarbia, P. Garcia, and F. Viadero, "Advanced model for the calculation of meshing forces in spur gear planetary transmissions," Meccanica, vol. 50, no. 7, pp. 1869-1894, 2015.

[4] Z. Chen, W. Zhai, Y. Shao, and K. Wang, "Mesh stiffness evaluation of an internal spur gear pair with tooth profile shift," Science China Technological Sciences, vol. 59, no. 9, pp. 13281339, 2016.

[5] A. Kahraman, "Planetary gear train dynamics," Journal of Mechanical Design, vol. 116, no. 3, pp. 713-720, 1994. 
[6] A. Kahraman, "Load sharing characteristics of planetary transmissions," Mechanism and Machine Theory, vol. 29, no. 8, pp. 1151-1165, 1994.

[7] R. G. Parker, "Physical explanation for the effectiveness of planet phasing to suppress planetary gear vibration," Journal of Sound and Vibration, vol. 236, no. 4, pp. 561-573, 2000.

[8] V. K. Ambarisha and R. G. Parker, "Suppression of planet mode response in planetary gear dynamics through mesh phasing," Journal of Vibration and Acoustics, vol. 128, no. 2, pp. 133-142, 2006.

[9] V. K. Ambarisha and R. G. Parker, "Nonlinear dynamics of planetary gears using analytical and finite element models," Journal of Sound and Vibration, vol. 302, no. 3, pp. 577-595, 2007.

[10] R. G. Parker and J. Lin, "Mesh phasing relationships in planetary and epicyclic gears," Journal of Mechanical Design, vol. 126, no. 2, pp. 365-370, 2004.

[11] Y. Chen and A. Ishibashi, "Investigation of noise and vibration of planetary gear drives," Gear Technology Magazine, vol. 23, no. 1, pp. 48-55, 2006.

[12] Y. Guo and R. G. Parker, "Analytical determination of mesh phase relations in general compound planetary gears," Mechanism and Machine Theory, vol. 46, no. 12, pp. 1869-1887, 2011.

[13] S. Y. Wang, M. N. Huo, C. Zhang et al., "Effect of mesh phase on wave vibration of spur planetary ring gear," European Journal of Mechanics A/Solids, vol. 30, pp. 820-827, 2011.

[14] S. H. Gawande, S. N. Shaikh, R. N. Yerrawar et al., "Noise level reduction in planetary gear set," Journal of Mechanical Design and Vibration, vol. 2, pp. 60-62, 2014.

[15] S. H. Gawande and S. N. Shaikh, "Experimental investigations of noise control in planetary gear set by phasing," Journal of Engineering, vol. 2014, Article ID 857462, 11 pages, 2014.

[16] F. Chaari, T. Fakhfakh, and M. Haddar, "Dynamic analysis of a planetary gear failure caused by tooth pitting and cracking," Journal of Failure Analysis and Prevention, vol. 6, no. 2, pp. 7378, 2006.

[17] G. Y. Li, F. Y. Li, Y. F. Wang, and D. Dong, "Fault diagnosis for a multistage planetary gear set using model-based simulation and experimental investigation," Shock and Vibration, vol. 2016, Article ID 9263298, 19 pages, 2016.

[18] G. Y. Li, F. Y. Li, H. H. Liu, and D. Dong, "Fault features analysis of a compound planetary gear set with damaged planet gears," Proceedings of the Institution of Mechanical Engineers, Part C: Journal of Mechanical Engineering Science, vol. 2017, Article ID 705906, 19 pages, 2017.

[19] Y. Hu, D. Talbot, and A. Kahraman, "A load distribution model for planetary gear sets," Journal of Mechanical Design, vol. 140, no. 5, Article ID 053302, 2018.

[20] B. Boguski, A. Kahraman, and T. Nishino, "A new method to measure planet load sharing and sun gear radial orbit of planetary gear sets," Journal of Mechanical Design, vol. 134, no. 7, 2012.

[21] H. Ligata, A. Kahraman, and A. Singh, "An experimental study of the influence of manufacturing errors on the planetary gear stresses and planet load sharing," Journal of Mechanical Design, vol. 130, no. 4, Article ID 041701, 2008. 


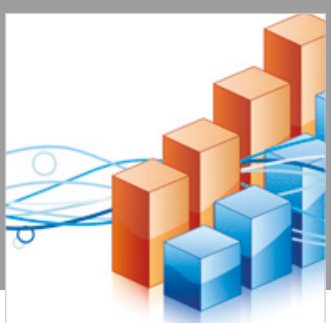

Advances in

Operations Research

\section{-n-m}
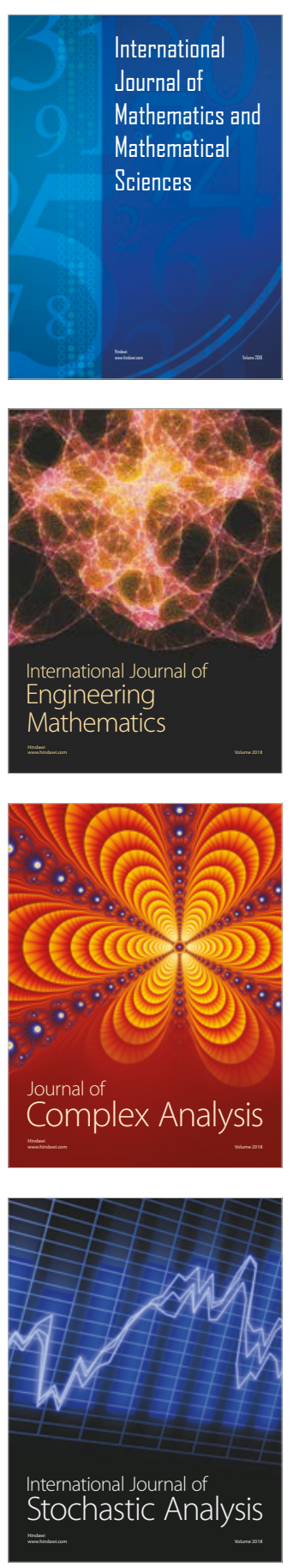
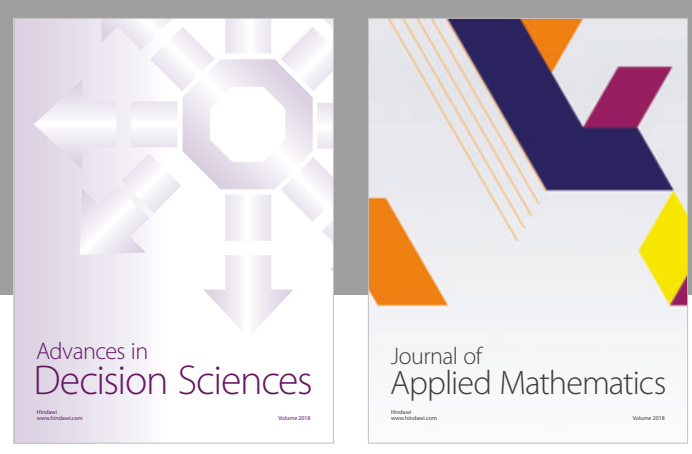

Journal of

Applied Mathematics
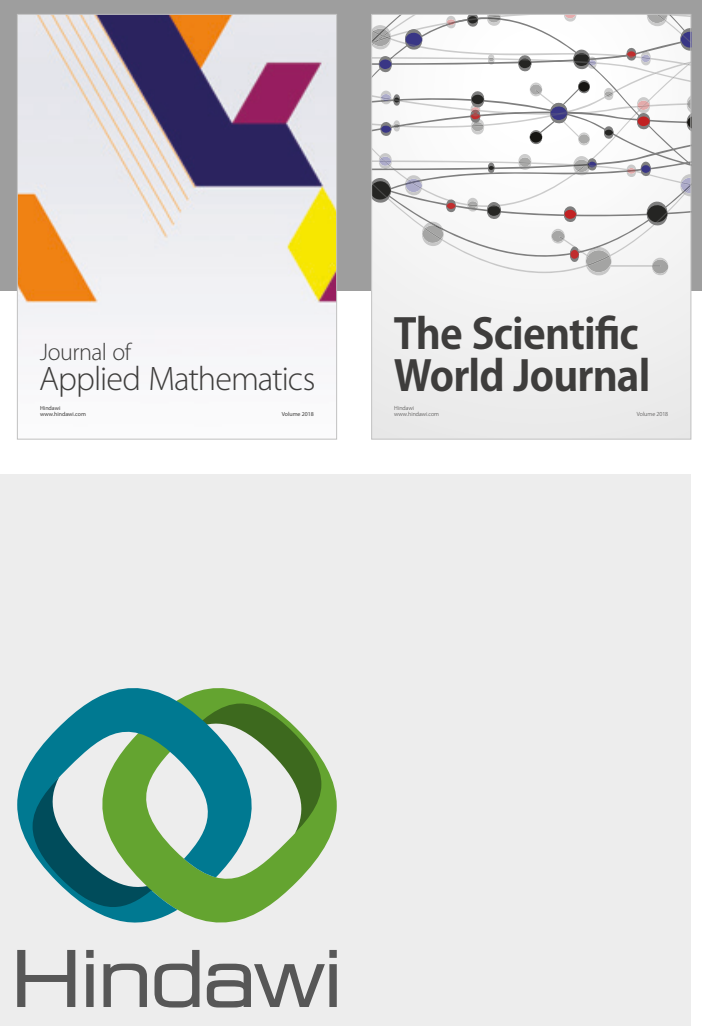

Submit your manuscripts at

www.hindawi.com

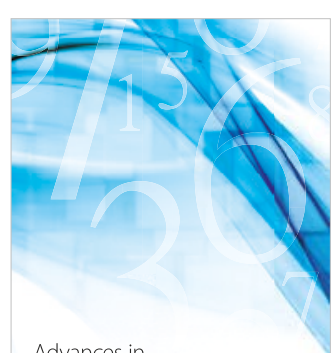

Advances in
Numerical Analysis
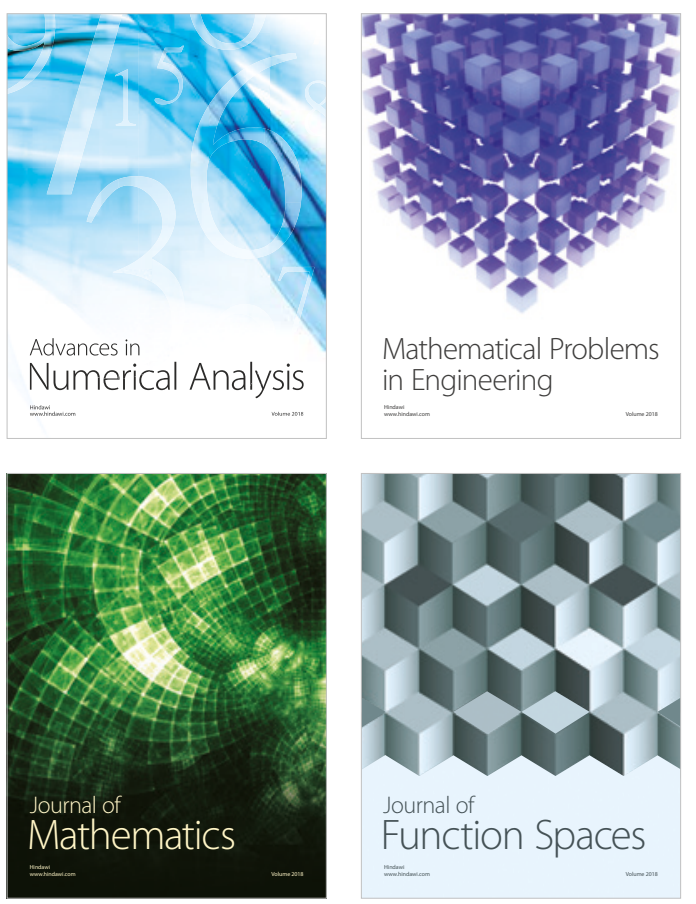

Mathematical Problems in Engineering

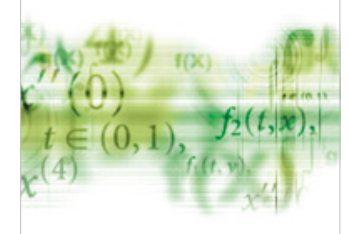

International Journal of

Differential Equations

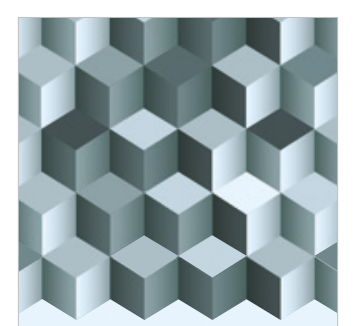

Journal of

Function Spaces

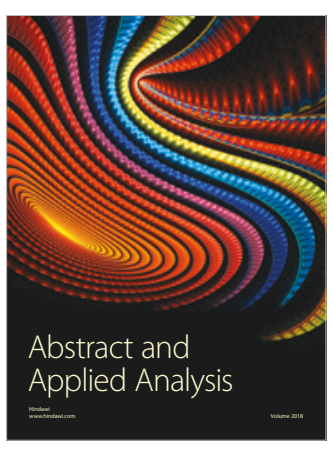

The Scientific

World Journal

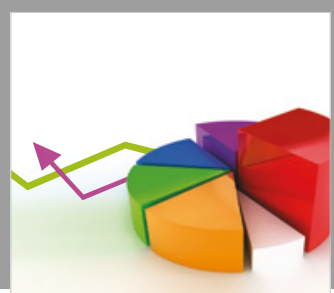

Journal of

Probability and Statistics
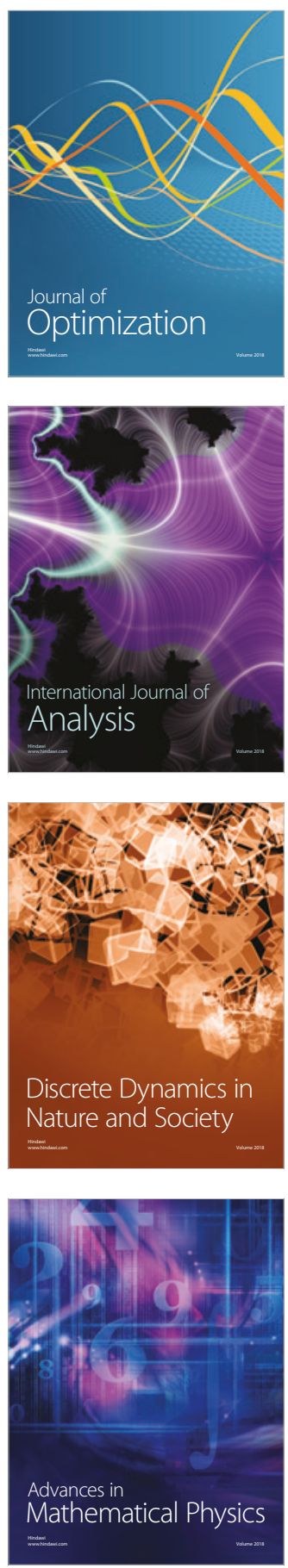